\title{
CORRIGENDUM
}

\section{Hydrodynamic damping of an oscillating cylinder at small Keulegan-Carpenter numbers - CORRIGENDUM}

\author{
Chengjiao Ren, Lin Lu, Liang Cheng and Tingguo Chen
}

doi:10.1017/jfm.2020.1159, Published by Cambridge University Press, 02 March 2021

The authors apologise that upon publication the funding information for The Australia Research Council Discovery Grant was incorrectly listed.

The correct Project ID is: DP200102804

The online version of this article has been updated

\section{REFERENCE}

Ren, C., Lu, L., Cheng, L. \& Chen, T. 2021 Hydrodynamic damping of an oscillating cylinder at small Keulegan-Carpenter numbers. J. Fluid Mech. 913, A36. doi:10.1017/jfm.2020.1159. 